\title{
Moderne medikamentöse Therapie der interstitiellen \\ Lungenerkrankung bei systemischer Sklerose
}

\section{Modern drug therapy for systemic sclerosis associated interstitial lung disease}

\section{(c) (i) $(9)$}

\section{Autoren}

Martin Aringer ${ }^{1}$, Dirk Koschel ${ }^{2,3}$, Andreas Krause ${ }^{4}$, Udo Schneider ${ }^{5}$, Sven Gläser ${ }^{6}$

Institute

1 Universitätsklinikum Carl Gustav Carus Dresden, Medizinische Klinik und Poliklinik III, Bereich Rheumatologie

2 Fachkrankenhaus Coswig, Lungenzentrum, Abteilung Innere Medizin und Pneumologie

3 Universitätsklinikum Carl Gustav Carus Dresden, Medizinische Klinik I, Bereich Pneumologie

4 Immanuel Krankenhaus Berlin, Abteilung Rheumatologie, Klinische Immunologie und Osteologie

5 Charité - Universitätsmedizin Berlin, Medizinische Klinik mit Schwerpunkt Rheumatologie und Klinische Immunologie

6 Vivantes Klinikum Neukölln und Spandau Berlin, Klinik für Innere Medizin - Pneumologie und Infektiologie sowie Universitätsmedizin Greifswald, Klinik und Poliklinik für Innere Medizin B

Schlüsselwörter

Systemische Sklerose, Interstitielle Lungenerkrankung, Therapie

Key words

systemic sclerosis, interstitial lung disease, therapy

online publiziert 03.12 .2021

Bibliografie

Dtsch Med Wochenschr 2022; 147: 179-186

DOI 10.1055/a-1658-5176

ISSN 0012-0472

(C) 2021. The Author(s).

This is an open access article published by Thieme under the terms of the Creative Commons Attribution-NonDerivative-NonCommercial License, permitting copying and reproduction so long as the original work is given appropriate credit. Contents may not be used for commecial purposes, or adapted, remixed, transformed or built upon. (https://creativecommons.org/licenses/by-nc-nd/4.0/)

Georg Thieme Verlag KG, Rüdigerstraße 14,

70469 Stuttgart, Germany
Korrespondenzadresse

Prof. Dr. Martin Aringer

Universitätsklinikum Carl Gustav Carus Dresden,

Fetscherstr. 74, 01307 Dresden, Deutschland

Martin.Aringer@uniklinikum-dresden.de

\section{ZUSAMMENFASSUNG}

Die systemische Sklerose mit interstitieller Lungenerkrankung (SSc-ILD) ist mit einem erhöhten Morbiditäts- und Mortalitätsrisiko verknüpft. Da es an zugelassenen Medikamenten mangelt, die den Krankheitsverlauf einer SSc-ILD effektiv beeinflussen, besteht ein hoher Bedarf an neuen Therapieoptionen. Die Behandlung mit immunmodulierenden Therapien sowie der autologen Stammzelltransplantation wird in klinischen Studien aktuell weiter untersucht. Kürzlich belegte zudem eine Phase-III-Studie die positive Wirkung des antifibrotischen Wirkstoffs Nintedanib auf den Verlust an Lungenvolumen und somit die Krankheitsprogression bei Patienten mit SSc-ILD. Eine Kombinationstherapie aus Nintedanib und Mycophenolat könnte basierend auf synergistischen Wirkprinzipien zukünftig einen ergänzenden Behandlungsansatz der SSc-ILD darstellen.

\section{ABSTRACT}

Systemic Sclerosis with Interstitial Lung Disease (SSc-ILD) is associated with an increased risk of morbidity and mortality. Because of a lack of approved medications that can effectively influence SSc-ILD disease course, there is a need for new therapeutic options. Treatment with immunomodulatory therapies as well as with autologous stem cell transplant is being further investigated in current clinical studies. Recently, a phase III study demonstrated the positive effect of the antifibrotic agent nintedanib on the loss of lung volume and thus disease progression in patients with SScILD. Due to its synergistic mechanism of action, combination therapy with nintedanib and mycophenolate could be a complementary treatment approach for SSc-ILD in the future. 


$\begin{array}{ll}\text { ABKÜRZUNGEN } \\ \text { AZA } & \text { Azathioprin } \\ \text { CRP } & \text { C-reaktives Protein } \\ \text { CYC } & \text { Cyclophosphamid } \\ \text { dcSSC } & \text { diffuse kutane SSc } \\ \text { DLco } & \text { Diffusionskapazität } \\ \text { FVC } & \text { forcierte Vitalkapazität } \\ \text { IPF } & \text { idiopathische Lungenfibrose } \\ \text { ICSSC } & \text { limitierte kutane SSc } \\ \text { MMF } & \text { Mycophenolat } \\ \text { mRSS } & \text { modified Rodnan Skin Score } \\ \text { RTX } & \text { Rituximab } \\ \text { SSC-ILD } & \text { Interstitielle Lungenbeteiligung der systemischen } \\ & \text { Sklerose } \\ \text { SSC } & \text { systemische Sklerose } \\ \text { TCZ } & \text { Tocilizumab } \\ \text { TLC } & \text { Totale Lungenkapazität }\end{array}$

\section{Hintergrund}

Die systemische Sklerose (SSc) ist eine seltene, heterogen verlaufende, oft lebensbedrohliche Kollagenose, die typischerweise mit der Fibrose der Haut und innerer Organe einhergeht [1, 2]. Etwa $60 \%$ der Patienten erkranken an limitierter kutaner SSc (IcSSc), 30 \% an diffuser kutaner SSc (dcSSc), die übrigen $10 \%$ leiden unter seltenen Subtypen [3]. Während sich die IcSSc meist schleichend entwickelt, zeichnet sich die dcSSc durch einen schnelleren Verlauf mit häufiger und rascher Organbeteiligung aus [3].

Bis zu 50 \% der SSc-Patienten entwickeln im Laufe der Erkrankung eine klinisch relevante interstitielle Lungenerkrankung (SSC-ILD). Die SSc-ILD ist für rund ein Drittel aller krankheitsassoziierten Todesfälle verantwortlich und zeichnet sich im Thorax-HRCT meist durch ein NSIP-ähnliches Muster mit Milchglaszeichnung, teils Retikulationen, Traktions-Bronchiektasen und subpleuralen Aussparungen aus [1,4]. Während Patienten im Frühstadium häufig asymptomatisch sind, äußert sich die SScILD später meist durch Belastungsdyspnoe, Erschöpfung und Reizhusten. Anhaltspunkte für die Entwicklung einer SSc-ILD sind neben dem Knisterrasseln im auskultatorischen Befund die Abnahme von Diffusionskapazität (DLco), forcierter Vitalkapazität (FVC) und totaler Lungenkapazität (TLC) sowie indirekt erhöhte Werte des C-reaktiven Proteins (CRP).

Das höchste Risiko zur Entwicklung einer ILD besteht im Anfangsstadium der SSc, daher sollte initial mittels Thorax-HRCT gescreent, die Lungenfunktion engmaschig kontrolliert und bei Verdacht auf Verschlechterung ein Verlaufs-CT durchgeführt werden [1]. Während gerade dcSSc-Patienten Symptome oft schnell entwickeln, zeigen andere einen milden, langfristig stabilen Krankheitsverlauf [1]. Die Progression der Erkrankung wird hierbei über die Verschlechterung im HRCT oder der Lungenfunktion definiert [1].

Typisch für die unbehandelte SSc-ILD ist eine progrediente Erkrankung mit Verlust von Lungenvolumen und einer hohen
Sterblichkeit [1, 4]. Die ILD spielt daher in der Prognose der SSc eine entscheidende Rolle. Insbesondere bei männlichen Personen und aktiven Rauchern besteht ein hohes Risiko einer raschen ILDProgression mit früher Sterblichkeit [5, 6]. Im Vergleich zu Patienten mit idiopathischer Lungenfibrose (IPF) verläuft die Krankheitsprogression allerdings im Mittelwert deutlich langsamer [7].

\section{Management der SSc-ILD}

Bis vor Kurzem gab es keine in Deutschland zugelassene Therapieoption für die SSc-ILD. Gesichert wurden nur immunmodulierende Ansätze in wenigen randomisierten Studien mit zudem begrenzter Effektstärke [3].

Das Ziel des vorliegenden Artikels besteht darin, einen Überblick über die aktuellen Therapieoptionen der SSc-ILD zu geben. Zu diesem Zweck wurde eine Literaturrecherche zu den zwischen 2006 und 2020 veröffentlichten klinischen Studien durchgeführt.

\section{Bisherige und aktuelle Behandlungsoptionen}

Historisch lag der Fokus in der Behandlung der SSc-ILD auf der Therapie mit ungesicherten immunmodulierenden Behandlungsansätzen, unter anderem Glukokortikoiden. Sie war jedoch mit einem 3-Jahres-Überleben von etwa $50 \%$ wenig erfolgreich. In den letzten Jahren hat sich die Therapie der SSc-ILD auf Basis klinischer Studien und durch Umsetzung pathophysiologischer Erkenntnisse erheblich gewandelt [8]. Kürzlich entwickelte ein europäisches Expertengremium nach Abschluss des Delphi-Prozesses erstmals evidenzbasierte Empfehlungen zur Einordnung der SSc-ILD ( $\triangleright$ Tab. 1) [9].

\section{Anti-inflammatorischer Therapieansatz}

\section{Cyclophosphamid}

Gemäß den noch aktuellen EULAR-Empfehlungen aus dem Jahr 2017 ist Cyclophosphamid (CYC) in der SSc-ILD die Therapie der Wahl. CYC führte oral verabreicht in der Scleroderma-Lung-Study (SLS I) zur signifikanten Verbesserung von Lungenfunktion, Dyspnoe, Hautverdickung und gesundheitsbezogener Lebensqualität ( $\triangleright$ Tab. 2) $[10,11]$. Nach Beendigung der einjährigen CYC-Therapie kam es jedoch erneut zu einer konsekutiven Verschlechterung, sodass eine immunmodulierende Erhaltungstherapie erforderlich. In einer zweiten Studie resultierte intravenös verabreichtes CYC in einer Verbesserung der FVC um 4,1\% (nicht statistisch signifikant, $\mathrm{P}=0,08)$.

\section{Mycophenolat}

Die Folgestudie SLS II verglich Mycophenolat (MMF) mit einem Jahr oral verabreichtem CYC und zeigte nach 2 Jahren in beiden Therapiearmen signifikante Verbesserungen von FVC und Mortalität [12]. MMF wies jedoch ein besseres Verträglichkeitsprofil auf ( $\triangleright$ Tab. 2) und kann im Gegensatz zu CYC längerfristig eingesetzt werden. 
- Tab. 1 Evidenz-basierte Empfehlungen zu Diagnostik und Management der SSc-ILD. Adaptiert nach [9].

\begin{tabular}{|l|l|}
\hline Merkmal & Empfehlung \\
\hline Risikofaktoren & Anti-Topoisomerase-I-Antikörper, männliches Geschlecht und eine dcSSc erhöhen das Risiko für die Entwicklung einer ILD. \\
\hline Screening & $\begin{array}{l}\text { Alle SSc-Patienten sollten einem ILD-Screening mittels HRCT und Lungenfunktionstests unterzogen werden. Die Häufigkeit } \\
\text { eines HRCT-Screenings sollte vom ILD-Risiko in Kombination mit klinischen Symptomen und der Lungenfunktion geleitet } \\
\text { werden. }\end{array}$ \\
\hline $\begin{array}{l}\text { Diagnose und Bestimmung } \\
\text { des Schweregrads }\end{array}$ & $\begin{array}{l}\text { Empfohlen wird der Einsatz des HRCT für die Diagnose und die Bestimmung der Schwere der SSc-ILD, unterstützt durch } \\
\text { Befunde aus Lungenfunktionstests und der klinischen Untersuchung. }\end{array}$ \\
\hline $\begin{array}{l}\text { Therapieeinleitung und } \\
\text { Behandlungsoptionen }\end{array}$ & $\begin{array}{l}\text { Bei allen Patienten mit schwerer oder progredienter SSc-ILD sollte eine Pharmakotherapie mit Mycophenolat, } \\
\text { Cyclophosphamid oder Nintedanib erwogen werden. Bei unbehandelten Patienten sollte ein engmaschiges Monitoring } \\
\text { auf Zeichen für eine Krankheitsprogression erfolgen. }\end{array}$ \\
\hline Krankheitsprogression & $\begin{array}{l}\text { Indikatoren einer Progression sind anhaltende Abnahme der Lungenfunktion, stärkere klinische Symptome und } \\
\text { Verschlechterung des HRCT in Ausmaß und/oder Muster der ILD. }\end{array}$ \\
\hline Therapie-Eskalation & $\begin{array}{l}\text { Bei Patienten mit progredienter Erkrankung oder unzureichendem Ansprechen sollte eine Therapie-Eskalation in Form } \\
\text { einer Dosismodifikation oder Änderung der pharmakologischen Behandlung mit Mycophenolat, Cyclophosphamid, } \\
\text { Nintedanib oder ggf. Rituximab erwogen werden. Die Eignung für eine Lungentransplantation sollte frühzeitig evaluiert } \\
\text { werden, insbesondere bei fortgeschrittener Erkrankung zum Diagnosezeitpunkt. Eine autologe hämatopoetische } \\
\text { Stammzelltransplantation (ASCT) kann bei sorgfältig ausgewählten Patienten in Betracht gezogen werden. }\end{array}$ \\
\hline
\end{tabular}

\section{Tocilizumab}

Die Phase-III-Studie „focuSSced“ untersuchte eine Therapie mit Tocilizumab (TCZ) bei Patienten mit aktiver, progredienter dcSSc mit inflammatorischem Phänotyp [13].

Obwohl eine signifikante Verbesserung des modifizierten Rodnan Skin-Scores (mRSS) nach 48 Wochen nicht erreicht wurde, resultierte TCZ in einer Überlegenheit gegenüber Placebo in Bezug auf FVC und gegenüber der Zeit bis zur Verschlechterung ( $\triangleright$ Tab. 2). In der Open-Label-Phase zeigten alle Patienten einen kontinuierlichen Rückgang des mRSS.

TCZ wurde von der US-Zulassungsbehörde FDA im Frühjahr 2021 für die SSc-ILD zugelassen.

\section{Rituximab}

Die B-Zell-Depletion mit Rituximab (RTX) wird auf Basis zweier kleiner kontrollierter Studien und offener Beobachtungsdaten als Off-Label-Rettungstherapie bei therapierefraktärer SSc-ILD verwendet $[14,15,28]$.

\section{Azathioprin}

Azathioprin (AZA) führte im Vergleich zu CYC in einer randomisierten Studie bei Patienten mit SSc-ILD zu einer signifikanten Verschlechterung der FVC- und DLco-Werte und wird daher für die Induktionstherapie nicht mehr in Betracht gezogen [16]. Es gibt aber positive, nicht kontrollierte Daten für die Erhaltungstherapie nach erfolgreicher CYC-Induktion, sodass diese Sequenz ähnlich wie beim systemischen Lupus erythematodes eine Option darstellt.

\section{Glukokortikoide}

Die lange praktizierte Therapie mit Glukokortikoiden in der SScILD ist aus heutiger Sicht obsolet. Glukokortikoide konnten keine Wirksamkeit für die SSc-ILD zeigen und erhöhen das Risiko einer lebensbedrohlichen renalen Krise erheblich. Eine gewisse Berechtigung haben niedrig dosierte Glukokortikoide hingegen für muskuloskelettale SSc-Manifestationen.

\section{Registerdaten zur Behandlungslage in Europa}

Von den im internationalen EUSTAR-Register gemeldeten Patienten mit SSc-ILD erhalten $71 \%$ immunmodulierende Medikamente, genauer

- 30,6\% Glukokortikoide (GC) in meist niedrig dosierter Monotherapie,

- $11,9 \%$ GC+CYC,

- $9,2 \%$ GC+AZA,

- $8,7 \%$ GC+Methotrexat,

- $7,3 \%$ GC+MMF

- und je etwa $5 \%$ CYC, MMF AZA oder MTX in Monotherapie ohne GC [17].

\section{Antifibrotischer Therapieansatz}

\section{Nintedanib}

Nintedanib ist für die Behandlung der IPF zugelassen und konnte die jährliche Abnahme der Lungenfunktion bei IPF etwa um die Hälfte reduzieren sowie den Krankheitsverlauf, auch in der Langzeitanwendung, bremsen ( $\triangleright$ Abb. 1 ). Zusammen mit positiven Daten in präklinischen SSc-ILD-Modellen war dies Anlass für die klinische Erprobung von Nintedanib zur Behandlung der SSc-ILD.

In der SENSCIS-Studie bei SSc-ILD führte Nintedanib im Vergleich zu Placebo nach 52 Wochen zu einem signifikant verringerten jährlichen FVC-Verlust ( $\triangleright$ Tab. 2) [7]. Die adjustierte jährliche FVC-Abnahme bei Patienten im Nintedanib-Arm betrug $44 \%$ [-52,4 ml/Jahr vs. -93,3 ml/Jahr unter Placebo, absolute Differenz 41,0 ml/Jahr (95\%-Kl: 2,9-79,0; $p=0,04)$ ] [7] ( Abb. 1, 2). Daten der offenen Verlängerungsstudie SENSCIS-ON bestätigen das Anhalten des Effekts von Nintedanib über mindestens 2 Jahre 

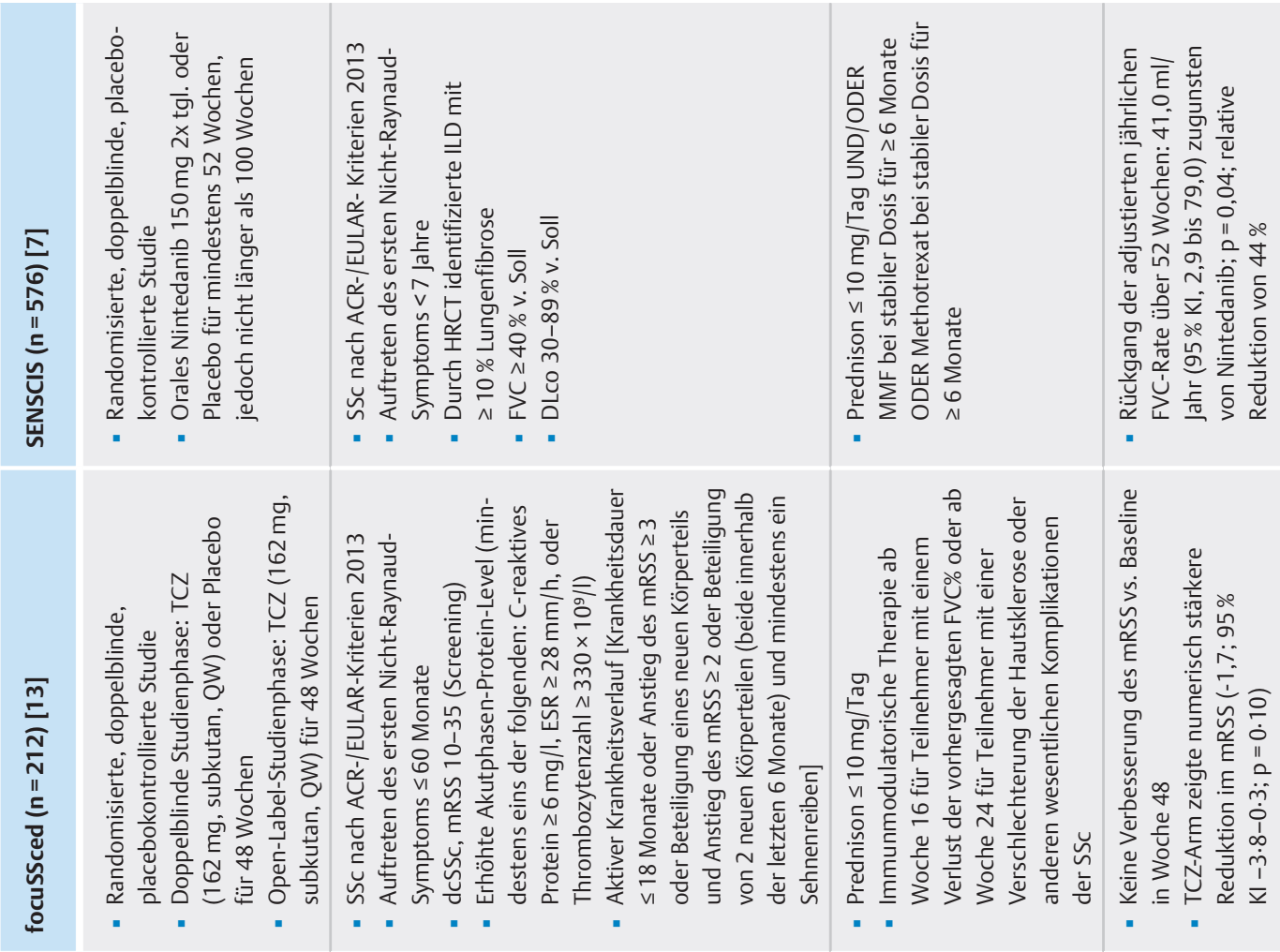

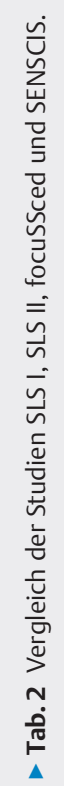
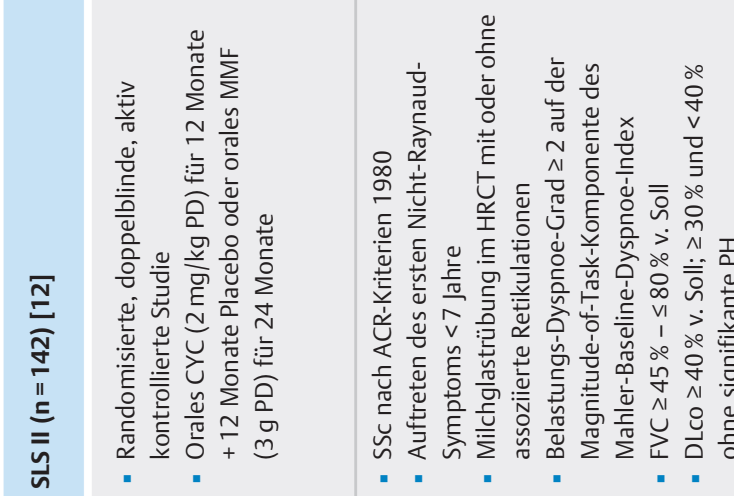

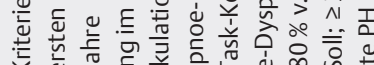

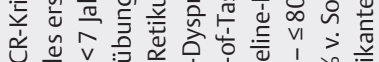

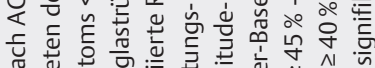

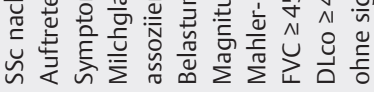

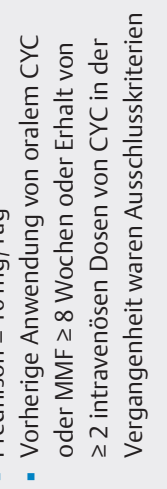

可

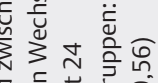

플

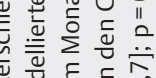

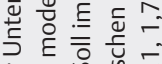

焉

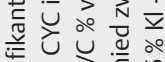

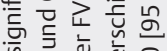

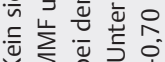

" "

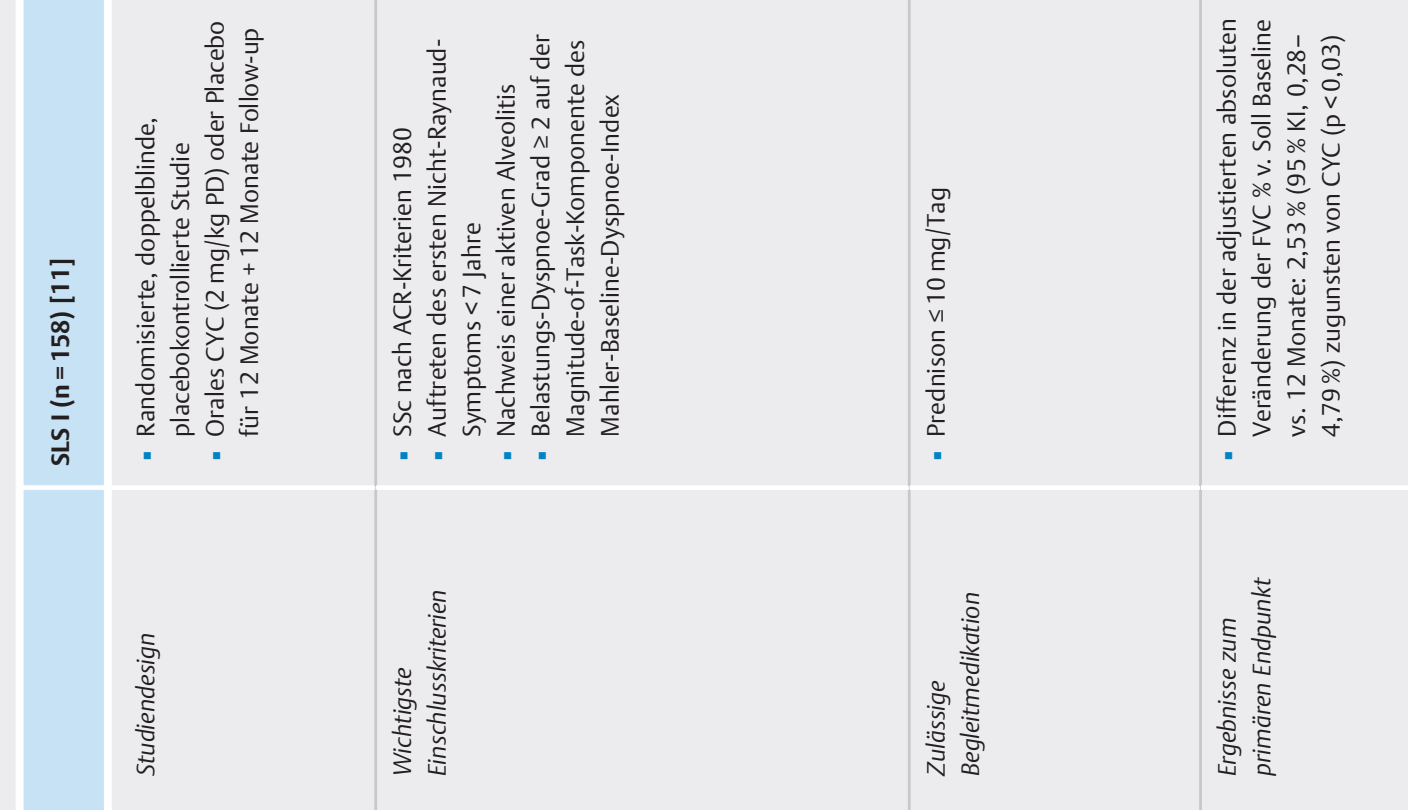




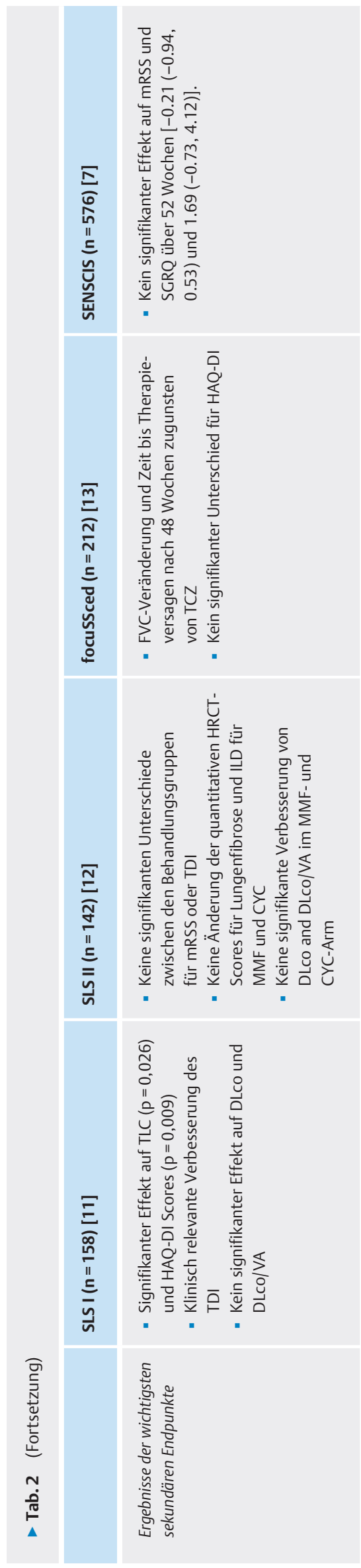

$[18,19]$. Es zeigte sich kein Effekt auf den mRSS. In post hoc durchgeführten Subanalysen reduzierte Nintedanib das Fortschreiten der ILD bei Patienten mit oder ohne MMF-Begleitmedikation, sowie unabhängig von SSc-Subtyp, ATA-Status, BMI, CRP, Ausmaß der ILD im HRCT, Einnahme von Kortikosteroiden, Gewichtsverlust und Geschlecht [7, 20-26]. Das Sicherheitsprofil von Nintedanib bei IPF und SSc-ILD war vergleichbar [7]. Die häufigsten Nebenwirkungen waren gastrointestinal, mild bis moderat ausgeprägt und meist gut handhabbar.

In der INBUILD-Studie bei Patienten mit chronischen progredient fibrosierenden ILDs ( $n=663)$, einschließlich 39 Patienten mit progredienter SSc-ILD, war die jährliche FVC-Abnahme im Nintedanib-Arm signifikant niedriger als unter Placebo [27]. Auch in der Subgruppenanalyse der SSc-ILD-Patienten zeigte Nintedanib eine konsistente Wirksamkeit [adjustierte Differenz (95\% KI) 120,7 mL $(-53,2,294,6), p=0,91]$.

Basierend auf den Ergebnissen der SENSCIS- und INBUILDStudien ist Nintedanib in der Therapie der SSC-ILD und bei chronischen progredient fibrosierenden ILDs von der Europäischen Arzneimittelkommission (EMA) sowie der FDA zugelassen.

Da Nintedanib eine neue Substanz darstellt, werden relevante Informationen in > Tab. 3 zusammengefasst.

\section{Kombination von Nintedanib und Immunsuppressiva}

In der SENSCIS-Studie nahm etwa die Hälfte der Patienten zu Studienbeginn zusätzlich MMF ein.

Diese SSc-Patienten mit MMF-Begleittherapie zeigten einen jährlichen FVC-Abfall von -40,2 ml unter Nintedanib und -66,5 ml unter Placebo; bei Patienten ohne MMF waren dies $-63,9 \mathrm{ml}$ unter Nintedanib und $-119,3 \mathrm{ml}$ unter Placebo (Interaktions-p-Wert: $p=0,452)$. Somit betrug die relative Reduktion der FVC unter Nintedanib in Kombination mit MMF $40 \%$ und ohne begleitende MMF-Therapie $46 \%$. Die Studie war aber nicht auf einen direkten Vergleich ausgelegt; Patienten mussten stabil (> 6 Monate) auf MMF eingestellt sein [7].

Denkbar ist hier eine Synergie der anti-inflammatorischen Wirkung durch MMF und der antifibrotischen Wirkung durch Nintedanib. Der potenzielle Nutzen der Kombination Nintedanib+MMF sollte daher in weiteren Studien untersucht werden. Eine signifikante Veränderung extrapulmonaler Manifestationen der SScILD, wie etwa der Fibrosierung der Haut, war durch Nintedanib in der SENSCIS-Studie nicht zu verzeichnen, sodass in vielen Fällen eine zusätzliche MMF-Therapie sinnvoll wäre, um das sonstige Erkrankungsgeschehen der SSc unter Kontrolle zu halten. Schwierig ist in diesem Zusammenhang der Off-Label-Status von MMF bei der Behandlung der SSc. In Kombination mit der aktuellen Off-Label-Rechtsprechung kann das dazu führen, dass SSc-ILDPatienten diese Medikamentenkombination verwehrt wird, obwohl sie aus unserer Sicht oft die höchste Erfolgsrate verspricht. 


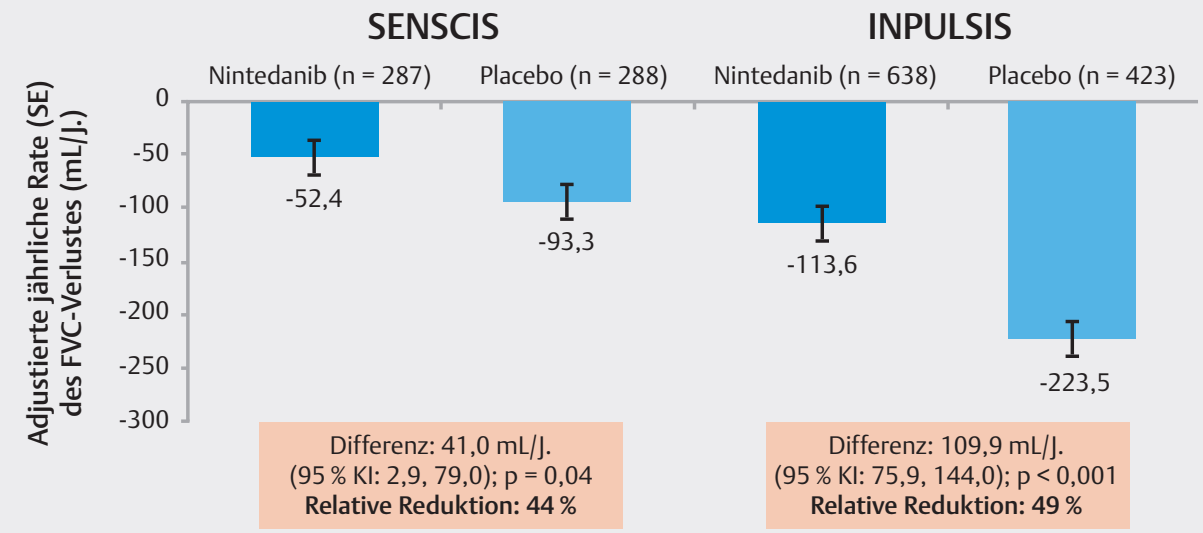

- Abb. 1 Jährliche Rate des FVC-Verlustes (ml/Jahr) in SENSCIS und INPULSIS. FVC= forcierte Vitalkapazität. Daten aus [7].

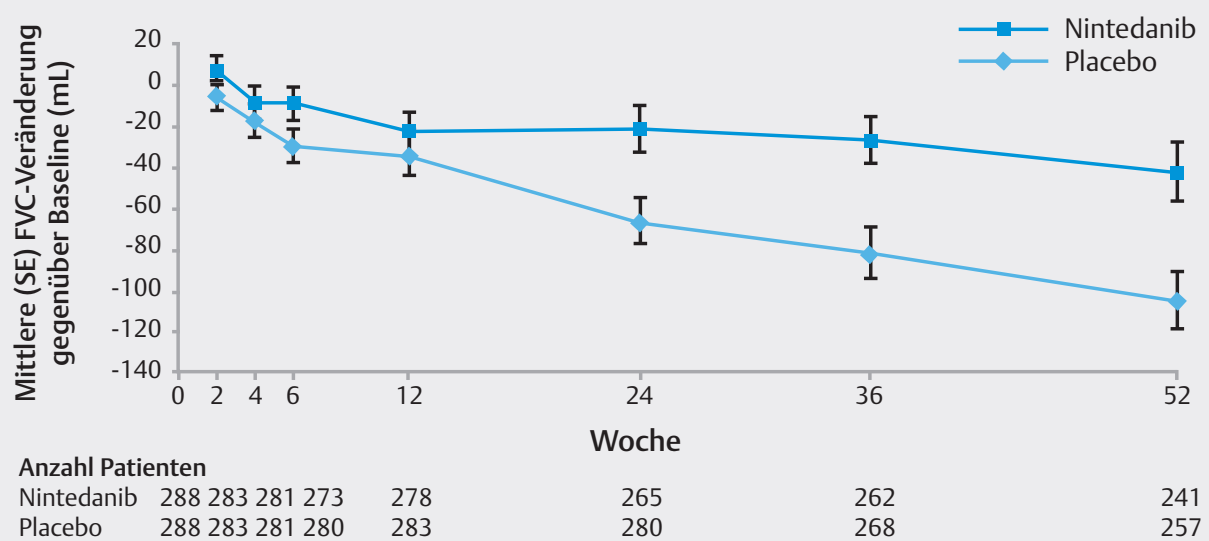

- Abb. 2 Veränderung der FVC vs. Baseline (ml) über 52 Wochen. FVC=forcierte Vitalkapazität. Daten aus [7].

Auch eine Kombination von Nintedanib und CYC in der Therapie der SSc-ILD wäre aufgrund der unterschiedlichen immunmodulierenden und antifibrotischen Angriffspunkte der Therapeutika durchaus denkbar, bedarf aber einer weiteren Untersuchung in klinischen Studien.

Einordnung der antifibrotischen Therapie in die Behandlungsalgorithmen

Neben der Immunmodulation steht mit der Zulassung von Nintedanib ein neues, über verschiedene Patientengruppen wirksames Therapieprinzip für die Behandlung der SSc-ILD zur Verfügung, welches die Therapieoptionen erweitert [7]. Im Einzelfall sollte die Frage „Immunmodulation und/oder Nintedanib-Gabe“ daher von Erkrankungsphase, individuellem Verlauf und Risikofaktoren abhängig gemacht werden. Studiendaten sprechen dafür, dass zumindest die Kombination Nintedanib und MMF sicher ist; es wurden keine signifikanten Einflüsse auf die Verträglichkeit gesehen. Im Zweifelsfall ist die Entscheidung im Rahmen interdisziplinärer Konferenzen in spezialisierten Zentren anzustreben.

\section{KERNAUSSAGEN}

- Die immunmodulierenden Off-Label-Therapieansätze Cyclophosphamid, Mycophenolat, Tocilizumab und Rituximab haben Wirksamkeit in der Behandlung der SSc-ILD gezeigt, sind aber individuell von variabler Effektivität.

- Glukokortikoide sind für die Behandlung der SSC-ILD nicht indiziert und erhöhen das Risiko der lebensbedrohlichen SSc-Nierenkrise.

- Nintedanib wirkt dem Verlust der Lungenfunktion gezielt entgegen und ist zur Behandlung der SSc-ILD zugelassen. Für die Therapie extrapulmonaler Manifestationen der SSc-ILD ist eine weitere Therapie notwendig.

- Basierend auf zwei unterschiedlichen Wirkprinzipien könnte die Kombinationstherapie aus Nintedanib und Mycophenolat zukünftig einen sich ergänzenden Behandlungsansatz der SSc-ILD darstellen. 
- Tab. 3 Praktische Aspekte zu Nintedanib in der Therapie der SSC-ILD.

\begin{tabular}{|c|c|}
\hline Anwendung & - Die empfohlene Dosis liegt bei 2-mal täglich 150 mg Nintedanib im Abstand von etwa 12 Stunden. \\
\hline $\begin{array}{l}\text { Nebenwirkungs- } \\
\text { management }\end{array}$ & $\begin{array}{l}\text { - Die häufigste Nebenwirkung ist Diarrhoe, die in Abhängigkeit der Schwere mit der Verordnung von Loperamid und } \\
\text { ggf. Dosisreduktion behandelt werden kann. Sollten bei schweren Durchfällen Opiate als Antidiarrhoika eingesetzt } \\
\text { werden, sollte deren Dosis und die Therapiedauer so gering wie möglich sein. }\end{array}$ \\
\hline Baseline Assessment & $\begin{array}{l}\text { - Vor Beginn einer Nintedanib-Therapie sollte bei allen Patienten die Leberfunktion getestet und bei Frauen im } \\
\text { gebärfähigen Alter eine Schwangerschaft mittels einem entsprechenden Test ausgeschlossen werden. }\end{array}$ \\
\hline \multirow[t]{3}{*}{ Besondere Personengruppen } & $\begin{array}{l}\text { Patienten mit Nierenfunktionsstörung } \\
\text { " Bei Patienten mit schwerer Nierenfunktionsstörung (<30 ml/min Kreatinin-Clearance) wurden Sicherheit und } \\
\text { Wirksamkeit von Nintedanib nicht untersucht. }\end{array}$ \\
\hline & $\begin{array}{l}\text { Patienten mit pulmonal-arterieller Hypertonie (PAH) } \\
\text { - Bei Patienten mit schwerer PAH wird die Gabe von Nintedanib derzeit nicht empfohlen. }\end{array}$ \\
\hline & $\begin{array}{l}\text { Patienten mit Leberfunktionsstörung } \\
\text { - Patienten mit leichter Leberfunktionsstörung (Child Pugh A) sollten nur mit einer reduzierten Dosis Nintedanib von } \\
100 \text { mg 2-mal täglich im Abstand von ca. } 12 \text { Stunden behandelt werden. } \\
\text { - Zur Sicherheit und Wirksamkeit von Nintedanib bei Patienten mit mittelschwerer und schwerer Leberfunktionsstörung } \\
\text { (Child Pugh B bzw. C) liegen keine Daten vor. Die Behandlung ist daher in dieser Patientengruppe nicht empfohlen. }\end{array}$ \\
\hline $\begin{array}{l}\text { Begleitmedikation und } \\
\text { Wechselwirkungen }\end{array}$ & $\begin{array}{l}\text { - Starke P-gp-Inhibitoren (z. B. Ketoconazol, Erythromycin oder Ciclosporin) können die Plasmaspiegel von Nintedanib } \\
\text { erhöhen. } \\
\text { - Starke P-gp-Induktoren (z. B. Rifampicin, Carbamazepin, Phenytoin oder Johanniskraut) können zur Verringerung des } \\
\text { Nintedanib-Plasmaspiegels führen. }\end{array}$ \\
\hline Patientengespräch & $\begin{array}{l}\text { Der Patient sollte explizit darüber aufgeklärt werden, dass die Therapie mit Nintedanib nicht die kurzfristige } \\
\text { Verbesserung von Symptomen, sondern den mittel- und langfristigen Erhalt größtmöglicher Teile der Lungenfunktion } \\
\text { zum gemeinsamen Ziel hat. }\end{array}$ \\
\hline
\end{tabular}

\section{Finanzielle Unterstützung}

Die vorliegende Publikation wurde durch finanzielle Unterstützung der Firma Boehringer Ingelheim Pharma GmbH \& Co. KG ermöglicht. Redaktionelle Unterstützung wurde durch die Firma Infill Healthcare Communication $\mathrm{GmbH}$ bereitgestellt.

\section{Interessenkonflikt}

M. Aringer: Honorare von und klinische Studien für Boehringer Ingelheim und Roche

D. Koschel: Honorare und Sponsoring von Boehringer Ingelheim und Roche

A. Krause: Honorare und Sponsoring von Boehringer Ingelheim, Honorare von Roche

U. Schneider: Honorare und Sponsoring von Boehringer Ingelheim und Roche

S. Gläser: Honorare und Sponsoring von Boehringer Ingelheim, Roche, Novartis, Berlin Chemie, Astra Zeneca

\section{Literatur}

[1] Cottin V, Brown KK. Interstitial lung disease associated with systemic sclerosis (SSc-ILD). Respir Res 2019; 20: 13. doi:10.1186/s12931-0190980-7

[2] Denton CP, Khanna D. Systemic sclerosis. Lancet Lond Engl 2017; 390 : 1685-1699. doi:10.1016/S0140-6736(17)30933-9

[3] Khanna D, Tashkin DP, Denton CP et al. Ongoing clinical trials and treatment options for patients with systemic sclerosis-associated interstitial lung disease. Rheumatology 2019; 58: 567-579. doi:10.1093/rheumatology/ key 151
[4] Wollin L, Distler JH, Denton CP et al. Rationale for the evaluation of nintedanib as a treatment for systemic sclerosis-associated interstitial lung disease. J Scleroderma Relat Disord 2019; 4: 212-218. doi:10.1177| 2397198319841842

[5] Takei R, Arita M, Kumagai S et al. Radiographic fibrosis score predicts survival in systemic sclerosis-associated interstitial lung disease. Respirology 2018; 23: 385-391. doi:10.1111/resp.13175

[6] Winstone TA, Assayag D, Wilcox PG et al. Predictors of mortality and progression in scleroderma-associated interstitial lung disease: a systematic review. Chest 2014; 146: 422-436. doi:10.1378/chest.13-2626

[7] Distler O, Highland KB, Gahlemann M et al. Nintedanib for Systemic Sclerosis-Associated Interstitial Lung Disease. N Engl J Med 2019; 380: 2518-2528. doi:10.1056/NEJMoa1903076

[8] Perelas A, Silver RM, Arrossi AV et al. Systemic sclerosis-associated interstitial lung disease. Lancet Respir Med 2020; 8: 304-320. doi:10.1016/S2213-2600(19)30480-1

[9] Hoffmann-Vold AM, Maher TM, Philpot EE et al. The identification and management of interstitial lung disease in systemic sclerosis: evidencebased European consensus statements. Lancet Rheumatol 2020; 2: e71e83. doi:10.1016/S2665-9913(19)30144-4

[10] Kowal-Bielecka O, Fransen ], Avouac ] et al. Update of EULAR recommendations for the treatment of systemic sclerosis. Ann Rheum Dis 2017; 76: 1327-1339. doi:10.1136/annrheumdis-2016-209909

[11] Tashkin DP, Elashoff R, Clements PJ et al. Cyclophosphamide versus placebo in scleroderma lung disease. N Engl J Med 2006; 354: 2655-2666. doi:10.1056/NEJMoa055120

[12] Tashkin DP, Roth MD, Clements PJ et al. Mycophenolate mofetil versus oral cyclophosphamide in scleroderma-related interstitial lung disease (SLS II): a randomised controlled, double-blind, parallel group trial. Lancet Respir Med 2016; 4: 708-719. doi:10.1016/S2213-2600(16)30152-7

[13] Khanna D, Lin CJF, Furst DE et al. Tocilizumab in systemic sclerosis: a randomised, double-blind, placebo-controlled, phase 3 trial. Lancet Respir Med 2020. doi:10.1016/S2213-2600(20)30318-0 
[14] Sircar G, Goswami RP, Sircar D et al. Intravenous cyclophosphamide vs rituximab for the treatment of early diffuse scleroderma lung disease: open label, randomized, controlled trial. Rheumatol Oxf Engl 2018; 57 : 2106-2113. doi:10.1093/rheumatology/key213

[15] Benad M, Koschel D, Herrmann K et al. Effects of cyclophosphamide and rituximab in patients with connective tissue diseases with severe interstitial lung disease. Clin Exp Rheumatol 2021

[16] Nadashkevich O, Davis P, Fritzler $\mathrm{M}$ et al. A randomized unblinded trial of cyclophosphamide versus azathioprine in the treatment of systemic sclerosis. Clin Rheumatol 2006; 25: 205-212. doi:10.1007/s10067-0051157-y

[17] Adler S, Huscher D. EUSTAR co-workers on behalf of the DeSScipher project research group within the EUSTAR network et al. Systemic sclerosis associated interstitial lung disease - individualized immunosuppressive therapy and course of lung function: results of the EUSTAR group. Arthritis Res Ther 2018; 20: 17. doi:10.1186/s13075-018-1517-z

[18] Allanore Y, Vonk MC, Azuma A et al. Continued Treatment with Nintedanib in Patients with Systemic Sclerosis-Associated Interstitial Lung Disease (SSC-ILD): Interim Analysis of SENSCIS-ON. Arthritis Rheumatol 2020; 72 (Suppl. 10)

[19] Maher TM, Distler O, Allanore Y et al. Effect of Nintedanib on Progression of Systemic Sclerosis-Associated Interstitial Lung Disease (SSc-ILD) Beyond 52 Weeks: Data from the SENSCIS Trial. C22 ILD Ther III 2020: A4558-A4558. doi:10.1164/ajrccm-conference.2020.201.1_MeetingAbstracts.A4558

[20] Highland K, Distler O, Kuwana M et al. Efficacy and safety of nintedanib in patients with systemic sclerosis-associated interstitial lung disease treated with mycophenolate: subgroup analysis of the SENSCIS trial. Lancet Respir Med 2020. doi:10.1016/S2213-2600(20)30330-1

[21] Goh N, Denton CP, Lynch DA et al. Effect of Nintedanib in Patients with Limited and Extensive Systemic Sclerosis-Associated Interstitial Lung Disease (SSc-ILD): Data from the SENSCIS Trial. A37 ILD Ther I 2020: A1525-A1525. doi:10.1164/ajrccm-conference.2020.201.1_MeetingAbstracts.A1525
[22] Jouneau S, Lescoat A, Riemekasten G et al. Efficacy and Safety of Nintedanib in Patients with Systemic Sclerosis-Associated Interstitial Lung Disease (SSC-ILD) by Body Mass Index (BMI) at Baseline: Subgroup Analysis of the SENSCIS Trial | A37. ILD THERAPY I. Am J Respir Crit Care Med 2020; 2020201A1526. Im Internet (Stand: 20.07.2020): https://www.atsjour nals.org/doi/abs/10.1164/ajrccm-conference.2020.201.1_MeetingAbs tracts.A1526

[23] Lescoat A, Jouneau S, Crestani B et al. Is the Rate of Lung Function Decline the Same in Patients with Systemic Sclerosis-Associated Ild (ssc-Ild) Who Experience Weight Loss? Data from the Senscis Trial. Ann Rheum Dis 2020; 79: 1110-1110. doi:10.1136/annrheumdis-2020eular.3535

[24] Riemekasten G, Carreira P, Saketkoo LA et al. Effects of nintedanib in patients with systemic sclerosis-associated ILD (SSC-ILD) and normal versus elevated C-reactive protein (CRP) at baseline: Analyses from the SENSCIS trial. Ann Rheum Dis 2020; 79: 413. doi:10.1136/annrheumdis2020-eular.3711

[25] Volkmann E, Vettori S, Varga J et al. Is There a Difference Between the Sexes in the Rate of Progression of Systemic Sclerosis-Associated Ild (ssc-Ild)? Data from the Senscis Trial. Ann Rheum Dis 2020; 79: 11181119. doi:10.1136/annrheumdis-2020-eular.2907

[26] Vonk MC, Distler O, Furst DE et al. Efficacy and Safety of Nintedanib in Patients with Systemic Sclerosis-Associated Interstitial Lung Disease: Subgroup Analysis of the SENSCIS Trial by Corticosteroid Use [abstract]. Arthritis Rheumatol 2019; 71 (Suppl. 10): 1643

[27] Flaherty KR, Wells AU, Cottin V et al. Nintedanib in Progressive Fibrosing Interstitial Lung Diseases. N Engl J Med 2019; 381: 1718-1727. doi:10.1056/NEJMoa1908681

[28] Ebata S, Yoshizaki A, Oba K et al. Lancet Rheumatology 2021; 3 (7): E489-E497 